\title{
Phenobarbital Versus Lorazepam for Management of Alcohol Withdrawal Syndrome: A Retrospective Cohort Study
}

Fadi Hawa ${ }^{1}$, Linsey Gilbert ${ }^{2}$, Benjamin Gilbert ${ }^{3}$, Vanessa Hereford ${ }^{3}$, Aya Hawa ${ }^{4}$, Alsadiq Al Hillan ${ }^{5}$, Mark Weiner ${ }^{6}$, Jeremy Albright ${ }^{7}$, Caleb Scheidel ${ }^{7}$, Ola Al-Sous ${ }^{1}$

1. Internal Medicine, St. Joseph Mercy Ann Arbor Hospital, Ann Arbor, USA 2. Internal Medicine/Palliative Care, St. Joseph Mercy Ann Arbor Hospital, Ann Arbor, USA 3. Internal Medicine, St. Joseph Mercy Livingston Hospital, Howell, USA 4. Pharmacy, St. Joseph Mercy Ann Arbor Hospital, Ann Arbor, USA 5. Internal Medicine/Hepatology, Baylor College of Medicine, Houston, USA 6. Internal Medicine/Addiction Medicine, St. Joseph Mercy Ann Arbor Hospital, Ann Arbor, USA 7. Statistics, St. Joseph Mercy Ann Arbor Hospital, Ann Arbor, USA

Corresponding author: Fadi Hawa, fadi.hawa@stjoeshealth.org

\section{Abstract}

\section{Introduction}

Annually, 500,000 episodes of alcohol withdrawal syndrome (AWS) are severe enough to require clinical attention. A symptom-triggered lorazepam regimen remains the standard of care for the management of hospitalized AWS patients. However, phenobarbital has also been shown to be an effective adjunctive therapy for severe AWS, reducing benzodiazepine use in the emergency department (ED) and the intensive care unit (ICU). The purpose of this study is to compare hospital length of stay (LOS) for AWS patients using phenobarbital-based versus lorazepam-based treatment protocols as monotherapy for management of AWS on general medical units.

\section{Methods}

This is a retrospective cohort study over a two-year period (March, 2016 to March, 2018), conducted at three hospitals within the St. Joseph Mercy Health System. We included 606 patients with a primary diagnosis of AWS or alcohol intoxication who met our inclusion criteria (543 in the lorazepam cohort and 63 in the phenobarbital cohort). Adjusted comparisons were done using propensity scoring methods. Hospital LOS was set as the primary outcome. Secondary outcomes included all-cause 30-day readmission, alcohol-related 30-day readmission, 30-day ED visits after discharge, and need for ICU transfer during hospital stay.

\section{Results}

Patients who received phenobarbital had a statistically significant shorter hospital LOS as compared to patients who received lorazepam $(2.8$ versus 3.6 days, $\mathrm{P}<0.001)$. Furthermore, the phenobarbital treatment group had statistically significant lower rates of all-cause 30 -day readmission $(11.11 \%$ versus $14.18 \%, \mathrm{P}=$ 0.020 ) and 30 -day ED visits after discharge ( $11.11 \%$ versus $18.6 \%, \mathrm{P}=0.015)$. No statistical significance was detected for alcohol-related 30-day readmission and the need for ICU transfer between the treatment groups.

\section{(c) Copyright 2021}

Hawa et al. This is an open access article distributed under the terms of the Creative Commons Attribution License CC-BY 4.0., which permits unrestricted use, distribution, and reproduction in any medium, provided the original author and source are credited.

\section{Conclusion}

This study suggests that phenobarbital may be a reasonable alternative to lorazepam in the management of AWS patients admitted to general medical units. Larger scale, well-executed, and adequately powered prospective studies and randomized controlled trials are needed to corroborate these findings.

Categories: Internal Medicine, Other

Keywords: alcohol misuse, alcohol withdrawal syndrome, alcohol addiction, phenobarbital, lorazepam, length of hospital stay (los), hospitalized patients

\section{Introduction}

Alcohol is the most widely available and abused substance in the United States. An estimated 1.2 million hospital admissions are related to alcohol abuse annually, and about 500,000 episodes of withdrawal symptoms are severe enough to require clinical attention annually [1]. Alcohol withdrawal syndrome (AWS) diagnosis is primarily made based on the Diagnostic and Statistical Manual of Mental Disorders (DSM-V) criteria [2].

The Clinical Institute Withdrawal Assessment for Alcohol Scale, Revised (CIWA-AR) is the most widely used scoring tool to guide symptom-triggered therapy [3]. This therapy method is beneficial in individualizing treatments, reducing both treatment duration and the amount of medications used, and is as efficacious as 
The currently accepted treatment regimens have varied but generally include both benzodiazepine (BZD) and non-BZD approaches [4]. Sedative-hypnotic drugs were recommended by the American Society of Addiction Medicine as the primary agents for managing alcohol withdrawal delirium [5]. Current evidence does not strongly indicate that a specific sedative-hypnotic agent is superior to others or that switching from one to another is helpful [5].

Benzodiazepines are most commonly used and recommended by addiction specialists because of their favorable therapeutic/toxic effect index [6]. The effectiveness of BZDs for the management of AWS has been demonstrated across multiple studies both in improving discomfort associated with acute withdrawal, and in decreasing the risk of progression to seizures and alcohol withdrawal delirium $[3,6]$. However, patients with chronic heavy alcohol use usually acquire tolerance to alcohol and can even develop cross-tolerance to BZDs [7]. Furthermore, BZDs may be associated with additional risks of oversedation, encephalopathy, and agitation in medically hospitalized patients. In addition, BZDs have been associated with an increased risk of rebound withdrawal symptoms and post-treatment drinking in multiple randomized controlled trials conducted in an ambulatory setting for AWS patients [8-12].

Barbiturates, such as phenobarbital have been proven to be clinically safe, cost-effective, and easy-to-use medications and can potentially be considered as alternatives to BZDs [13,14]. They have different binding properties and receptor affinity when compared to BZDs that are believed to result in a reduced crosstolerance between phenobarbital and alcohol $[15,16]$. Moreover, commonly used doses of phenobarbital for AWS have not been associated with clinically significant sedation [17].

Recent literature supports the similarity between phenobarbital and BZDs with regard to tolerability and effectiveness in the treatment of AWS [17,18]. Despite these trends, there is only modest evidence comparing hospital length of stay (LOS) as a primary outcome between phenobarbital and BDZs in management of AWS patients admitted to general medical units in acute-care hospital setting [17-21].

Within our health system (St. Joseph Mercy Health System) which is comprised of five Michigan hospitals, there is a CIWA-AR dose-based driven protocol in place that allows physicians to choose different pharmaceutical options and routes of administration for management of AWS. The two existing options within this protocol are lorazepam and phenobarbital, orally, intramuscularly or intravenously. The choice of medication is mainly driven by the treating physician's preference. In general medical units, different trends towards which medication used have been noted among these hospitals within the same health system. However, they all share the practice of using phenobarbital if a patient is transferred to the intensive care unit (ICU). Given the observed internal variance within our health system and lack of quality data regarding AWS management, we compared the two protocols employed across three of our health system hospitals (Hospital A, Hospital B, and Hospital C).

Our study aimed to compare hospital LOS for AWS patients using phenobarbital-based versus lorazepambased treatment protocols as monotherapy for the management of AWS on general medical units. We hypothesized no significant difference in hospital LOS between phenobarbital and lorazepam. This article was previously presented as a meeting abstract at the 2020 AAAP Annual Scientific Meeting on December 8, $2019[22]$.

\section{Materials And Methods}

\section{Study design}

This is a retrospective cohort study for patients who were admitted to general medical units of three hospitals (Hospital A, Hospital B, and Hospital C) within the same health system (St. Joseph Mercy Health System) with a primary diagnosis of alcohol intoxication or AWS over a two-year period from March, 2016 to March, 2018. Hospital A is a 537-bed tertiary teaching hospital with a case-mix index-adjusted length of stay (CMI-ALOS) of 3.06, Hospital B is a 136-bed teaching hospital with CMI-ALOS of 2.57, and Hospital C is a 133-bed teaching hospital with CMI-ALOS of 2.49. CMI-ALOS is defined as the ratio of the number of days of hospital care that were utilized to care for patients adjusted for the documented severity of the illnesses [23].

Hospital LOS was defined as the primary outcome for the study. Secondary outcomes included, need for ICU transfer, all-cause 30-day readmission rate, alcohol-related 30-day readmission rate (both inpatient and observation statuses were included as a readmission event), and 30-day emergency department (ED) visits after discharge.

This study was intended to be a pilot observational study based on the small cohort of patients, given the low prevalence of alcohol withdrawal in the inpatient population and the need for a large sample size to power a non-inferiority study.

\section{Inclusion and exclusion criteria}


Eligible patients were adults aged 18-100 years without sex discrimination, who were admitted for alcohol intoxication or withdrawal to any of the above-defined facilities. Patients were excluded if they were admitted directly to ICU, pregnant women, prisoners, and patients who were transferred from an outside facility, or received an addiction medicine specialist consultation.

\section{Variables}

Data were obtained retrospectively through accessing the electronic medical records post-hospitalization. Data was abstracted both electronically and manually. Double data abstraction was used for manual abstractors until inter-rater reliability was achieved. Data was then reviewed using the inclusion and exclusion criteria. Collected variables included general demographics (gender, age, and ethnicity), major medical comorbidities (seizure disorder, coronary artery disease, hypertension, diabetes mellitus, chronic kidney disease, chronic obstructive pulmonary disease, and liver disease), AWS-related variables (initial CIWA score, and maximum CIWA score), outcome-related variables (hospital LOS, need for ICU transfer, alcohol-related 30-day readmission rate, all-cause 30-day readmission rate, and 30-day ED visits after discharge). Further variables that could have potentially influenced treatment decision or outcome were collected, which included presence or absence of urine drug screen, seizure incidence during hospitalization, depression, anxiety, schizophrenia, schizoaffective disorder, bipolar disorder, discharge to inpatient psychiatry unit, and concomitant use of gabapentin.

\section{Statistical analysis}

Unadjusted comparisons for all covariates and study outcomes by treatment type were assessed using chisquare or Fisher exact tests for categorical data and t-tests or Mann-Whitney tests for interval-level variables. Adjusted comparisons were conducted using propensity score methods. Specifically, propensity scores (the probability of receiving each treatment) were estimated based on a model in which all covariates are entered as predictors. The propensity scores were then converted to weights, and the tests were repeated using the propensity score-weighted data. Propensity score weights were preferable to matching for determining adjusted treatment effects given that no observations were discarded. All analyses were completed using R Version 3.6.0 [24], assuming a significance level of 0.05 .

\section{Ethical consideration}

The study was approved by the St. Joseph Mercy Health System Institutional Review Board. Informed consent was waived given the retrospective nature of the study.

\section{Results}

A total of 1,007 charts for patients admitted with a diagnosis of alcohol intoxication or withdrawal during the time period March, 2016 to March, 2018 were reviewed. After applying the exclusion criteria, 606 patients were included in the study (543 in the lorazepam cohort and 63 in the phenobarbital cohort) (Figure $1)$.

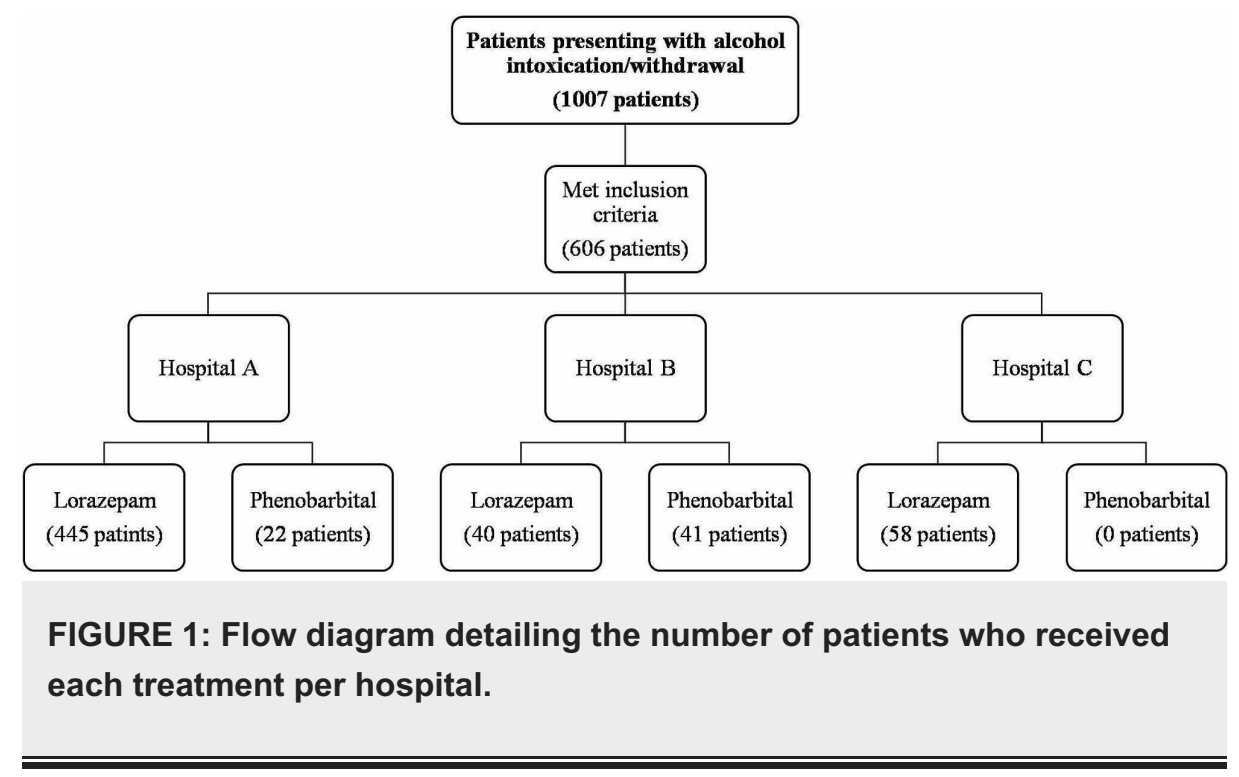

Table 1 demonstrates baseline demographics and clinical characteristics among treatment groups. The treatment groups are balanced by all confounders except for initial CIWA score and presence of urine drug screen before propensity score weighing. After weighing the treatment groups by propensity scores, the groups are balanced by all confounders except for chronic kidney disease (CKD), which is likely due to the fact that the phenobarbital treatment group had zero patients with CKD (Table 1). 


\section{Cureus}

\begin{tabular}{|c|c|c|c|c|c|}
\hline Variable & Label & Lorazepam $(n=543)$ & Phenobarbital $(n=63)$ & Unadjusted P-value & PS weighted P-value \\
\hline \multicolumn{6}{|l|}{ Categorical variables, $\mathrm{n}(\%)$} \\
\hline \multirow[t]{3}{*}{ Sex } & & & & 0.546 & 0.919 \\
\hline & Female & $173(31.86 \%)$ & $23(36.51 \%)$ & & \\
\hline & Male & $370(68.14 \%)$ & $40(63.49 \%)$ & & \\
\hline \multirow[t]{4}{*}{ Ethnicity } & & & & $>0.999$ & 0.622 \\
\hline & Black & $38(7 \%)$ & $4(6.35 \%)$ & & \\
\hline & White & $502(92.45 \%)$ & $59(93.65 \%)$ & & \\
\hline & Declined & $3(0.55 \%)$ & $0(0 \%)$ & & \\
\hline \multirow[t]{4}{*}{ Hospital } & & & & $<0.001$ & $<0.001$ \\
\hline & Hospital A & 445 (81.95\%) & $22(34.92 \%)$ & & \\
\hline & Hospital B & $40(7.37 \%)$ & $41(65.08 \%)$ & & \\
\hline & Hospital C & $58(10.68 \%)$ & $0(0 \%)$ & & \\
\hline Anxiety & & $216(39.78 \%)$ & $27(42.86 \%)$ & 0.737 & 0.767 \\
\hline Bipolar disorder & & $75(13.81 \%)$ & $9(14.29 \%)$ & $>0.999$ & 0.701 \\
\hline Coronary artery disease & & $22(4.05 \%)$ & $2(3.17 \%)$ & $>0.999$ & 0.67 \\
\hline Depression & & $201(37.02 \%)$ & $23(36.51 \%)$ & $>0.999$ & 0.541 \\
\hline Diabetes mellitus & & $35(6.45 \%)$ & $2(3.17 \%)$ & 0.412 & 0.493 \\
\hline Hypertension & & $241(44.38 \%)$ & $27(42.86 \%)$ & 0.923 & 0.827 \\
\hline Liver disease & & $133(24.49 \%)$ & $18(28.57 \%)$ & 0.579 & 0.698 \\
\hline Schizoaffective disorder & & $7(1.29 \%)$ & $1(1.59 \%)$ & 0.587 & 0.828 \\
\hline Schizophrenia & & $13(2.39 \%)$ & $2(3.17 \%)$ & 0.663 & 0.867 \\
\hline Chronic kidney disease & & $11(2.03 \%)$ & $0(0 \%)$ & 0.615 & 0.009 \\
\hline Chronic obstructive pulmonary disease & & $43(7.92 \%)$ & $3(4.76 \%)$ & 0.461 & 0.302 \\
\hline Seizure incidence during hospitalization & & $10(1.86 \%)$ & $1(1.64 \%)$ & $>0.999$ & 0.991 \\
\hline Urine drug screen & & $339(62.43 \%)$ & $51(80.95 \%)$ & 0.006 & 0.104 \\
\hline Use of gabapentin & & $159(29.28 \%)$ & $18(28.57 \%)$ & $>0.999$ & 0.962 \\
\hline Discharge to inpatient psychiatry unit & & $37(6.81 \%)$ & $4(6.35 \%)$ & $>0.999$ & 0.644 \\
\hline \multirow[t]{7}{*}{ Blood alcohol level } & & & & 0.865 & 0.906 \\
\hline & $<10$ & $133(26.55 \%)$ & $15(25.86 \%)$ & & \\
\hline & $10-100$ & $72(14.37 \%)$ & $8(13.79 \%)$ & & \\
\hline & $101-200$ & $63(12.57 \%)$ & $6(10.34 \%)$ & & \\
\hline & $201-300$ & $87(17.37 \%)$ & $14(24.14 \%)$ & & \\
\hline & $301-400$ & $95(18.96 \%)$ & $9(15.52 \%)$ & & \\
\hline & $>400$ & $51(10.18 \%)$ & $6(10.34 \%)$ & & \\
\hline \multicolumn{6}{|l|}{ Continuous variables, mean (SD) } \\
\hline Age & & 47.178 (12.742) & 45.568 (13.695) & 0.377 & 0.666 \\
\hline Initial CIWA score & & $9.046(5.87)$ & $11.016(7.54)$ & 0.049 & 0.859 \\
\hline Max CIWA & & .073) & $16.286(6.566)$ & 0.666 & 0.29 \\
\hline
\end{tabular}




\section{Cureus}

TABLE 1: Patients' baseline demographics and clinical characteristics - all hospitals $(n=606)$.

Propensity score weighing is used to balance treatment groups by all confounders. PS: propensity score; CIWA: Clinical Institute Withdrawal Assessment.

\begin{tabular}{|c|c|c|c|c|}
\hline & \multicolumn{4}{|c|}{$\begin{array}{l}\text { Comparing the two treatment groups across the three hospitals and after adjusting for baseline demographi } \\
\text { and clinical variables, patients in the phenobarbital treatment group had a statistically significant shorter } \\
\text { mean LOS when compared to patients in the lorazepam treatment group ( } 2.8 \text { versus } 3.6 \text { days, } \mathrm{P}<0.001 \text { ). In } \\
\text { addition, patients in the phenobarbital treatment group had a statistically significant lower all-cause } 30 \text {-day } \\
\text { readmission rate }(11.11 \% \text { versus } 14.18 \%, \mathrm{P}=0.020 \text { ) and } 30 \text {-day ED visits after discharge }(11.11 \% \text { versus } \\
18.6 \%, \mathrm{P}=0.015 \text { ). There was no statistical significance detected for alcohol-related } 30 \text {-day readmission and } \\
\text { need for ICU transfer between the treatment groups (Table } 2 \text { ). }\end{array}$} \\
\hline Primary and secondary outcomes & Lorazepam $(n=543)$ & Phenobarbital ( $n=63$ ) & Unadjusted P-value & PS weighted P-value \\
\hline \multicolumn{5}{|l|}{ Categorical outcomes, $\mathrm{N}(\%)$} \\
\hline Transferred to ICU & $38(7.04 \%)$ & $2(3.23 \%)$ & 0.416 & 0.114 \\
\hline All-cause 30-day readmission & $77(14.18 \%)$ & $7(11.11 \%)$ & 0.635 & 0.020 \\
\hline Alcohol-related 30-day readmission & $65(11.97 \%)$ & $6(9.52 \%)$ & 0.715 & 0.045 \\
\hline ED visit within 30 days of discharge & $101(18.6 \%)$ & $7(11.11 \%)$ & 0.195 & 0.015 \\
\hline \multicolumn{5}{|l|}{ Continuous outcomes, mean (SD) } \\
\hline Los (days) & $3.664(2.324)$ & $2.805(1.255)$ & $<0.001$ & $<0.001$ \\
\hline
\end{tabular}

\section{TABLE 2: Primary and secondary outcomes - all hospitals $(n=606)$.}

Propensity score weighing is used to balance treatment groups by all confounders. ED: emergency department; ICU: intensive care unit; LOS: length of stay; PS: propensity score.

Hospital B was noted to have a relatively equal patient distribution among treatment groups. Accordingly, a subgroup analysis for Hospital B was performed. The treatment groups are balanced by all confounders except for anxiety before propensity score weighing. After weighing the treatment groups by propensity scores, the groups are balanced by all confounders (Table 3). 


\section{Cureus}

\begin{tabular}{|c|c|c|c|c|c|}
\hline Variable & Label & Lorazepam $(n=40)$ & Phenobarbital $(n=41)$ & Unadjusted P-value & PS weighted P-value \\
\hline \multicolumn{6}{|l|}{ Categorical variables, $n(\%)$} \\
\hline \multirow[t]{3}{*}{ Sex } & & & & 0.753 & 0.693 \\
\hline & Female & $16(40 \%)$ & $14(34.15 \%)$ & & \\
\hline & Male & $24(60 \%)$ & $27(65.85 \%)$ & & \\
\hline \multirow[t]{2}{*}{ Ethnicity } & & & & - & - \\
\hline & White & $40(100 \%)$ & $41(100 \%)$ & & \\
\hline Anxiety & & $11(27.5 \%)$ & $22(53.66 \%)$ & 0.03 & 0.41 \\
\hline Bipolar disorder & & $1(2.5 \%)$ & $5(12.2 \%)$ & 0.201 & 0.085 \\
\hline Coronary artery disease & & $0(0 \%)$ & $1(2.44 \%)$ & $>0.999$ & 0.327 \\
\hline Depression & & $13(32.5 \%)$ & $15(36.59 \%)$ & 0.878 & 0.794 \\
\hline Diabetes mellitus & & $1(2.5 \%)$ & $0(0 \%)$ & 0.494 & 0.293 \\
\hline Hypertension & & $14(35 \%)$ & $16(39.02 \%)$ & 0.885 & 0.712 \\
\hline Liver disease & & $9(22.5 \%)$ & $13(31.71 \%)$ & 0.495 & 0.533 \\
\hline Schizoaffective disorder & & $0(0.00 \%)$ & $0(0.00 \%)$ & $\cdot$ & $\cdot$ \\
\hline Schizophrenia & & $0_{(0 \%)}$ & $1(2.44 \%)$ & $>0.999$ & 0.341 \\
\hline Chronic kidney disease & & $0(0.00 \%)$ & $0(0.00 \%)$ & - & - \\
\hline Chronic obstructive pulmonary disease & & $10(25 \%)$ & $3(7.32 \%)$ & 0.062 & 0.109 \\
\hline Seizure incidence during hospitalization & & $0(0 \%)$ & $1(2.56 \%)$ & 0.494 & 0.328 \\
\hline Urine drug screen & & $32(80 \%)$ & $35(85.37 \%)$ & 0.73 & 0.381 \\
\hline Use of gabapentin & & $18(45 \%)$ & $12(29.27 \%)$ & 0.217 & 0.229 \\
\hline Discharge to inpatient psychiatry unit & & $3(7.5 \%)$ & $1(2.44 \%)$ & 0.359 & 0.193 \\
\hline \multirow{7}{*}{ Blood alcohol level } & & & & 0.746 & 0.996 \\
\hline & $<10$ & $8(20.51 \%)$ & $9(24.32 \%)$ & & \\
\hline & 10-100 & $8(20.51 \%)$ & $5(13.51 \%)$ & & \\
\hline & $101-200$ & $4(10.26 \%)$ & $3(8.11 \%)$ & & \\
\hline & $201-300$ & $6(15.38 \%)$ & $10(27.03 \%)$ & & \\
\hline & 301-400 & $7(17.95 \%)$ & $7(18.92 \%)$ & & \\
\hline & $>400$ & $6(15.38 \%)$ & $3(8.11 \%)$ & & \\
\hline \multicolumn{6}{|l|}{ Continuous variables, mean (SD) } \\
\hline ge & & 44.154 (11.911) & 43.723(10.914) & 0.866 & 0.832 \\
\hline Initial CIWA score & & $11.625(7.11)$ & $12.61(8.053)$ & 0.561 & 0.819 \\
\hline Max CIWA & & $17.6(6.484)$ & $16.878(6.29)$ & 0.613 & 0.615 \\
\hline
\end{tabular}

TABLE 3: Baseline demographics and clinical characteristics - Hospital B $(n=81)$.

Propensity score weighing is used to balance treatment groups by all confounders. CIWA: Clinical Institute Withdrawal Assessment; PS: propensity score.

Patients receiving either lorazepam or phenobarbital at Hospital B were then compared for primary and secondary outcomes after adjusting for baseline demographic and clinical variables. There was no statistical 
significance detected between treatment groups in regards to hospital LOS, need for ICU transfer or 30-day readmission rate. However, the phenobarbital treatment group had a statistically significant lower rate for 30-day ED visits after discharge when compared to patients in the lorazepam treatment group (14.63\% versus $35 \%, \mathrm{P}=0.017$ ) (Table 4 ).

\begin{tabular}{|c|c|c|c|c|}
\hline Sub-group analysis outcome (Hospital B) & Lorazepam $(n=40)$ & Phenobarbital $(n=41)$ & Unadjusted P-value & PS weighted P-value \\
\hline \multicolumn{5}{|l|}{ Categorical outcomes, $\mathrm{n}(\%)$} \\
\hline Transferred to ICU & $3(7.5 \%)$ & $1(2.5 \%)$ & 0.615 & 0.267 \\
\hline All-cause 30-day readmission & $5(12.5 \%)$ & $2(4.88 \%)$ & 0.264 & 0.188 \\
\hline Alcohol-related 30-day readmission & $4(10 \%)$ & $2(4.88 \%)$ & 0.432 & 0.353 \\
\hline ED visit within 30 days of discharge & $14(35 \%)$ & $6(14.63 \%)$ & 0.062 & 0.017 \\
\hline \multicolumn{5}{|l|}{ Continuous outcomes, mean (SD) } \\
\hline LoS (Days) & $2.999(1.103)$ & $2.689(1.138)$ & 0.218 & 0.200 \\
\hline
\end{tabular}

TABLE 4: Primary and secondary outcomes - Hospital $B(n=81)$.

Propensity score weighing is used to balance treatment groups by all confounders. ED: emergency department; ICU: intensive care unit; LOS: length of stay; PS: propensity score.

\section{Discussion}

This is a pilot retrospective cohort study which compared the use of phenobarbital and lorazepam in the management of patients with AWS admitted to general medical units. The study included patients from three hospitals within the same health system. These hospitals have relatively similar CMI-ALOS and also share very similar workflow standards and treatment protocols. In this study, phenobarbital had a statistically significant shorter hospital LOS when compared to lorazepam. Furthermore, the phenobarbital treatment group had a statistically significant lower all-cause 30-day readmission rates and 30-day ED visits after discharge. Other secondary outcomes including alcohol-related 30-day readmission rate and need for ICU transfer showed no statistically significant difference.

A subgroup analysis for Hospital B was conducted given a relatively equal patient distribution across treatment groups (41 patients in the phenobarbital cohort and 40 patients in the lorazepam cohort). This has revealed no statistically significant difference in hospital LOS between treatment groups. Secondary outcomes were only statistically significant for a lower rate of ED visits within 30 days of discharge for phenobarbital when compared to lorazepam.

The propensity scoring method was done to ensure adjusted comparisons between the treatment groups especially for factors that may play a role in the management of AWS. Psychiatric disorders, inpatient psychiatry disposition, substance use disorders, or concomitant use of gabapentin are factors that might have either influenced the treatment decision or confounded the studied outcomes. Although the treatment groups were mostly balanced prior to propensity score weighing, weights were still used in the analysis to ensure comparable treatment groups based on these factors.

Our study results are consistent with the available literature and add to the growing body of evidence that phenobarbital may be a potentially feasible alternative to lorazepam in management of AWS. To our knowledge, this is the first study comparing hospital LOS as a primary outcome between lorazepam and phenobarbital in the treatment of AWS patients admitted to general medical units.

The safety and utility of phenobarbital in management of AWS as a monotherapy or as an adjunct to BZD, has been evaluated across multiple clinical settings [19-21,25-27]. Mo et al. reviewed a group of studies including 4 prospective controlled and 3 retrospective clinical trials to assess the efficacy and safety of barbiturates with or without BZDs versus BZDs for the treatment of AWS in the acute setting [17]. None of the studies reviewed demonstrated the inferiority of barbiturates to BZD in the management of AWS. Furthermore, overall safety profiles of barbiturates were comparable to those of BZDs across all studies included. Hammond et al., evaluated patient outcomes associated with phenobarbital use with or without BZDs for AWS by reviewing four controlled trials and five observational studies [18]. These authors concluded that phenobarbital may have a role in AWS treatment alongside BZDs or as monotherapy. Furthermore, patients with severe AWS who received phenobarbital required less escalation of their care, and those with mild to moderate AWS spent less time in the ED and did not require further care following discharge. These findings were suggestive of similar or even improved outcomes with phenobarbital when 
compared to alternative therapies, including BZDs. Unfortunately, both of these systematic reviews lacked high-quality evidence and contained a high degree of heterogeneity of the included studies, preventing the performance of a meta-analysis.

Two randomized controlled trials conducted in an ED setting evaluated the outcomes of phenobarbital for AWS management. The first is a placebo-controlled trial that studied the initial level of hospital admission (ICU versus telemetry versus floor wards) [19]. The trial showed that patients receiving a single dose of intravenous phenobarbital had a decreased ICU admission rate (phenobarbital versus placebo, 8\% versus $25 \%$ with $95 \%$ confidence interval [4\%-32\%]). No statistical differences were noted in admission neither to telemetry or floor wards nor in median ICU or total hospital LOS. Furthermore, there was no difference in the incidence of adverse outcomes between phenobarbital and placebo. The second trial compared intravenous phenobarbital to intravenous lorazepam in the treatment of acute AWS with regard to efficacy to improve symptoms during ED visits and at 48-hour reassessment as the primary outcome [20]. There was no statistical difference between phenobarbital and lorazepam in baseline CIWA scores $(\mathrm{P}=0.3)$, at ED discharge $(\mathrm{P}=0.4)$, or at 48 -hour re-evaluation $(\mathrm{P}=0.7)$. Therefore, phenobarbital was found to be as effective as lorazepam in the treatment of acute AWS.

Nisavic et al. conducted a retrospective review to evaluate the development of alcohol withdrawal-related complications following the initiation of treatment with either phenobarbital or BZD [21]. No statistical significance was detected between the two treatment protocols from a primary clinical outcome standpoint including alcohol-related seizure activity, hallucination, or delirium. Furthermore, no difference was detected in any of the study's secondary outcomes including hospital LOS, ICU admission rate and LOS, medication-related adverse events, or discharge against medical advice. Thus, offering further evidence that phenobarbital, like BZDs, appears to be a well-tolerated and effective treatment for AWS.

The main limitation of our study is that the population comes from three hospitals with a relatively unbalanced number of patients in each treatment group (543 in the lorazepam cohort and 63 in the phenobarbital cohort). This creates an inherited bias that cannot be controlled for as it is related to the treating physician's preference to a specific treatment protocol (phenobarbital versus lorazepam) in addition to the individual hospital workflow. However, all three hospitals share relatively comparable CMI-ALOS and are part of the same health system which probably reduces the weight of this limitation. For that reason, the subgroup analysis for Hospital B was performed given the relatively equal patient distribution among treatment groups, which showed no significant difference in hospital LOS between phenobarbital and lorazepam. Nonetheless, these results continue to be in concordance with the available literature suggesting that phenobarbital may be a reasonable alternative to BZDs. Furthermore, there are limits to the study's generalizability as the majority of patients included were white, which is reflective of the three hospitals' local community demographics. Other limitations to the study include its retrospective nature, lack of randomization, and lack of knowledge whether patients were readmitted to other health systems within 30 days after discharge.

\section{Conclusions}

In conclusion, this pilot retrospective cohort study suggests that phenobarbital may be a reasonable alternative to lorazepam in management of AWS patients admitted to general medical units. When taking into account the BZDs-related adverse events including oversedation, encephalopathy, agitation, and increased risk of rebound withdrawal symptoms, phenobarbital may represent a reasonable alternative with a potential for improved outcomes. Larger scale, well-executed, and adequately powered prospective studies and randomized controlled trials are needed to provide conclusive evidence to support the non-inferiority of phenobarbital to BZDs as a treatment option for AWS.

\section{Additional Information \\ Disclosures}

Human subjects: Consent was obtained or waived by all participants in this study. St. Joseph Mercy Health System Institutional Review Board issued approval R-18-1795. The study was approved by the St. Joseph Mercy Health System Institutional Review Board. Informed consent was waived given the retrospective nature of the study. Animal subjects: All authors have confirmed that this study did not involve animal subjects or tissue. Conflicts of interest: In compliance with the ICMJE uniform disclosure form, all authors declare the following: Payment/services info: All authors have declared that no financial support was received from any organization for the submitted work. Financial relationships: All authors have declared that they have no financial relationships at present or within the previous three years with any organizations that might have an interest in the submitted work. Other relationships: All authors have declared that there are no other relationships or activities that could appear to have influenced the submitted work.

\section{Acknowledgements}

We acknowledge the following individuals for assisting in this project: Richard Shellenberger, DO; Amy Burghardt, MS, MPH; and Anupam Suneja, MD. 


\section{References}

1. Gortney JS, Raub JN, Patel P, Kokoska L, Hannawa M, Argyris A: Alcohol withdrawal syndrome in medical patients. Cleve Clin J Med. 2016, 83:67-79. 10.3949/ccjm.83a.14061

2. American Psychiatric Association: Diagnostic and Statistical Manual of Mental Disorders . American Psychiatric Association, Washington, DC; 2013. http://www.psychiatry.org/psychiatrists/practice/dsm.

3. Saitz R, Mayo-Smith MF, Roberts MS, Redmond HA, Bernard DR, Calkins DR: Individualized treatment for alcohol withdrawal. A randomized double-blind controlled trial. JAMA. 1994, 272:519-523. 10.1001/jama.1994.03520070039035

4. Schuckit MA: Recognition and management of withdrawal delirium (delirium tremens) . N Engl J Med. 2014, 371:2109-2113. 10.1056/NEJMra1407298

5. Mayo-Smith MF, Beecher LH, Fischer TL, et al.: Management of alcohol withdrawal delirium. An evidencebased practice guideline. Arch Intern Med. 2004, 164:1405-1412. 10.1001/archinte.164.13.1405

6. Mayo-Smith MF: Pharmacological management of alcohol withdrawal. A meta-analysis and evidence-based practice guideline. JAMA. 1997, 278:144-151. 10.1001/jama.278.2.144

7. Sanna E, Mostallino MC, Busonero F, et al.: Changes in GABA(A) receptor gene expression associated with selective alterations in receptor function and pharmacology after ethanol withdrawal. J Neurosci. 2003, 23:11711-11724. 10.1523/JNEUROSCI.23-37-11711.2003

8. Malcolm R, Myrick H, Roberts J, Wang W, Anton RF, Ballenger JC: The effects of carbamazepine and lorazepam on single versus multiple previous alcohol withdrawals in an outpatient randomized trial. J Gen Intern Med. 2002, 17:349-355. 10.1046/j.1525-1497.2002.10201.x

9. Myrick H, Malcolm R, Randall PK, Boyle E, Anton RF, Becker HC, Randall CL: A double blind trial of gabapentin vs. lorazepam in the treatment of alcohol withdrawal. Alcohol Clin Exp Res. 2009, 33:15821588. 10.1111/j.1530-0277.2009.00986.x

10. Malcolm R, Myrick H, Roberts J, Wang W, Anton RF: The differential effects of medication on mood, sleep disturbance, and work ability in outpatient alcohol detoxification. Am J Addict. 2002, 11:141-50. 10.1080/10550490290087910

11. Malcolm R, Myrick LH, Veatch LM, Boyle E, Randall PK: Reported sleep, sleepiness, and repeated alcohol withdrawals: a randomized, double blind, controlled comparison of lorazepam vs gabapentin. J Clin Sleep Med. 2007, 3:24-32.

12. Poulos CX, Zack M: Low-dose diazepam primes motivation for alcohol and alcohol-related semantic networks in problem drinkers. Behav Pharmacol. 2004, 15:503-512. 10.1097/00008877-200411000-00006

13. Kwan P, Brodie MJ: Phenobarbital for the treatment of epilepsy in the 21st century: a critical review . Epilepsia. 2004, 45:1141-1149. 10.1111/j.0013-9580.2004.12704.x

14. Ives TJ, Mooney 3rd AJ, Gwyther RE: Pharmacokinetic dosing of phenobarbital in the treatment of alcohol withdrawal syndrome. South Med J. 1991, 84:18-21. 10.1097/00007611-199101000-00006

15. Leggio L, Kenna GA, Swift RM: New developments for the pharmacological treatment of alcohol withdrawal syndrome. A focus on non-benzodiazepine GABAergic medications. Prog Neuropsychopharmacol Biol Psychiatry. 2008, 32:1106-1117. 10.1016/j.pnpbp.2007.09.021

16. Teichberg VI, Tal N, Goldberg O, Luini A: Barbiturates, alcohols and the CNS excitatory neurotransmission: specific effects on the kainate and quisqualate receptors. Brain Res. 1984, 291:285-292. 10.1016/00068993(84)91260-5

17. Mo Y, Thomas MC, Karras GE: Barbiturates for treatment of alcohol withdrawal syndrome: a systematic review of clinical trials. J Crit Care. 2016, 32:101-107. 10.1016/j.jcrc.2015.11.022

18. Hammond DA, Rowe JM, Wong A, Wiley TL, Lee KC, Kane-Gill SL: Patient outcomes associated with phenobarbital use with or without benzodiazepines for alcohol withdrawal syndrome: a systematic review. Hosp Pharm. 2017, 52:607-616. 10.1177/0018578717720310

19. Rosenson J, Clements C, Simon B, et al.: Phenobarbital for acute alcohol withdrawal: a prospective randomized double-blinded placebo-controlled study. J Emerg Med. 2013, 44:592-598.e2. 10.1016/j.jemermed.2012.07.056

20. Hendey GW, Dery RA, Barnes RL, Snowden B, Mentler P: A prospective, randomized, trial of phenobarbital versus benzodiazepine for acute alcohol withdrawal. Am J Emerg Med. 2011, 29:382-385. 10.1016/j.ajem.2009.10.010

21. Nisavic M, Nejad SH, Isenberg BM, et al.: Use of phenobarbital in alcohol withdrawal management - a retrospective comparison study of phenobarbital and benzodiazepines for acute alcohol withdrawal management in general medical patients. Psychosomatics. 2019, 60:458-467. 10.1016/j.psym.2019.02.002

22. Hawa F, Gilbert L, Gilbert B, Hereford V, Weiner M, Al Sous O: Phenobarbital vs lorazepam for alcohol withdrawal syndrome: a retrospective cohort study. Am J Addict. 2020, 29:216-216.

23. Mendez CM, Harrington DW, Christenson P, Spellberg B: Impact of hospital variables on case mix index as a marker of disease severity. Popul Health Manag. 2014, 17:28-34. 10.1089/pop.2013.0002

24. Tippmann S: Programming tools: adventures with R. Nature. 2015, 517:109-110. 10.1038/517109a

25. Nguyen TA, Lam SW: Phenobarbital and symptom-triggered lorazepam versus lorazepam alone for severe alcohol withdrawal in the intensive care unit. Alcohol. 2020, 82:23-27. 10.1016/j.alcohol.2019.07.004

26. Oks M, Cleven KL, Healy L, et al.: The safety and utility of phenobarbital use for the treatment of severe alcohol withdrawal syndrome in the medical intensive care unit. J Intensive Care Med. 2020, 35:844-850. 10.1177/0885066618783947

27. Ibarra F Jr: Single dose phenobarbital in addition to symptom-triggered lorazepam in alcohol withdrawal . Am J Emerg Med. 2020, 38:178-181. 10.1016/j.ajem.2019.01.053 